\title{
A Line Inhomogeneity in an Elastic Half Plane Under Anti-Plane Shear Loading
}

\author{
George, N, Emenogu \\ Department of Mathematics Michael Okpara University Of Agriculture, Umudike, Abia State, Nigeria
}

\begin{abstract}
An elastic homogeneous isotropic material with a right line inhomogeneity embedded in the material under Anti-shear is analyzed; the mathematical model of the problem is a boundary value problem formulated using the mellin transform and solved by the Wiener-Hoph Techniques. A closed form solution for displacement is obtained from which the stress intensity factor is calculated. The stress field were found to have square-root singularity at the inner tip. As a result of this, micro-cracking can initiate at the inner tip of the line inhomogeneity in the matrix depending on the applied loads. The outer tip showed no singularly.
\end{abstract}

\section{Introduction}

One of the basic problems in solid mechanics is that of determining the elastic fields in a loaded homogeneous/heterogeneous medium. Inhomogeneities whether wanted or unwanted or deliberately introduced may drastically alter the elastic response of a material as well as its plastic and fracture properties.

A rigid line inhomogeneity embedded in an elastic material is of theoretical interest because it is the counterpart of conventional crack in solids.

According to a near tip asymptotic expansion, we know that the stress field have a square-root singularity at the inhomegeneity tip. Fracture mechanics analyses for a line inhomegeneity has been carried out by many researchers $[1,2]$ for various configurations in infinite and semi-infinite medium with loading at infinity.

The fields are usually modeled by a boundary value problem for laplace equation in two dimensions. This has often been solved by conformal mapping, integral transforms, perturbation methods, numerical techniques and ergen value techniques among the techniques.

All the work in the literature led to fracture criteria such as stress intensity factors which are in line with the linear fracture mechanics for cracks and crack initiation direction from the tip of the line inhomogeneity depending on the applied loads.

In this work, we have investigated bodies with finite boundaries under anti-plane shear with point loading. The mellin transform and all wiener Hoph techniques has been used to solve the boundary value problem.

The closed form solutions for the displacement and stress fields have been obtained. The stress fields at the inhomogeneity tips were obtained and found to have square root singularity at the inner tip. The mode III stress intensity factor was also determine $[3,4]$

\section{Mathematical formulation}

The problem is to determine the stress state in an elastic homogeneous solid material occupying the region expressed in cylindrical polar coordinates $(r, \theta, z)$

$$
\begin{aligned}
-\infty<z<\infty ; \quad x= & r \cos \theta ; \quad y=r \sin \theta \\
& -\pi / 2 \leq \theta \geq \frac{\pi}{2} \text { and } r \leq \alpha
\end{aligned}
$$

With a rigid line inhomogeneity which is imbedded in the elastic material in the region

$$
y=0 \text {, and } 0 \leq x \leq \alpha
$$

The material is isotropic and is subjected to anti-plane shear deformation arising from a pair of concentrated loads $T$ and $Q$ applied at distances 1 and $\mathrm{h}$ from the origin as shown in fig. 1.

Introducing polar coordinates $(r, \theta)$, the $z$ component of the displacement denoted by $W(r, \theta)$ satisfies the governing equation.

$W_{r r}+\frac{1}{r} W_{r}+\frac{1}{r^{2}} W_{\theta \theta}=0 ; \quad r \geq 0 \quad-\pi / 2 \leq \theta \geq \frac{\pi}{2}$

The non-vanishing polar stresses are

$$
\begin{aligned}
& \sigma \theta_{z}(r, \theta)=\frac{\mu}{r} \frac{\partial}{\partial \theta} W(r, \theta) \\
& \sigma r_{z}(r, \theta)=\mu \frac{\partial}{\partial r} W(r, \theta)
\end{aligned}
$$

The boundary conditions are 


$$
\begin{aligned}
& W(r, \theta)=0 \quad 0 \leq r \leq \alpha \\
& \sigma_{\theta} Z\left(r, \frac{\pi}{2}\right)=T \delta(r-l) \\
& \sigma_{\theta} Z\left(r, \frac{\pi}{2}\right)=Q \delta(r, h)
\end{aligned}
$$

The asymptotic behavior of the stresses are;

$$
\sigma_{\theta z} ; \sigma_{r z}=\left\{\begin{array}{l}
0\left(r^{-\lambda}\right) ; 0<\lambda 1 / 2 \text { as } r \rightarrow 0 \\
0\left(r^{-1}\right) \text { as } r \rightarrow \infty \\
0(r-a)^{-1 / 2} \text { as } r \rightarrow \text { a and } \theta \rightarrow 0
\end{array}\right.
$$

The continuity conditions of tractions and displacements are

$$
\begin{array}{lc}
W\left(r, 0^{+}\right)=\left(r, 0^{-}\right)=0 & 0 \leq r \leq a \\
W\left(r, 0^{+}\right)=\left(r, 0^{-}\right)= & r \geq a \\
\sigma_{\theta z}\left(r, 0^{+}\right)=\sigma_{\theta z}\left(r, 0^{-}\right)= & r \geq a
\end{array}
$$

Using mellin transform defined by

$$
\bar{W}(S, \theta)=\int_{0}^{\infty} W(r, \theta) r^{s-1} d r
$$

(1) is transformed to

$$
\left(\frac{d^{2}}{d \theta^{2}}+S^{2}\right) \bar{W}(S, \theta)=0 \quad \lambda-1<\operatorname{Res}<0 ;-\pi / 2 \leq \theta \leq \pi / 2
$$

Transforming the boundary conditions, we have

$$
\begin{aligned}
& \bar{W}(S, 0)=a^{S} \bar{V}(S), \text { where } \\
& \bar{V}(S)=\int_{1}^{\infty} W(a \tau, 0) \tau^{s-1} d \tau \\
& \frac{d \bar{W}}{d \theta}(S, \pi / 2)=\frac{1}{\mu} T l^{S} \\
& \frac{d \bar{W}}{d \theta}(S,-\pi / 2)=\frac{1}{\mu} Q h^{s} \\
& \frac{d \bar{W}}{d \theta}\left(S, 0^{+}\right)-\frac{d \bar{W}}{d \theta}\left(S, 0^{-}\right)=\frac{1}{\mu} a^{s} \bar{u}^{s}(s)
\end{aligned}
$$

We consider the solution of (10) of the form

$$
\bar{W}(S, \theta)= \begin{cases}A_{1}(S) \cos \theta S+ & B_{1}(S) \sin \theta S, \\ A_{2}(S) \cos \theta S+ & B_{2}(S) \sin \theta S,\end{cases}
$$

Where $A_{1}(S) ; \quad B_{1}(S) ; A_{2}(S)$ and $B_{2}(S)$ are to be determined from the boundary conditions. Now using

$\bar{W}\left(S, 0^{+}\right)=\bar{W}(S, 0)=a^{S} \bar{V}(S)$,

We have

$A_{1}(S)=A_{2}(S)=a^{S} \bar{V}(S)$,

$\frac{d \bar{W}}{d \theta}= \begin{cases}-S A_{1}(S) \sin \theta S+S B_{1}(S) \cos \theta S ; & 0 \leq \theta \leq \pi / 2 \\ -S A_{2}(S) \sin \theta S+S B_{2}(S) \cos \theta S ; & \pi / 2 \leq \theta \leq 0\end{cases}$

$\frac{d \bar{W}}{d \theta}\left(S, 0^{+}\right)-\frac{d \bar{W}}{d \theta}\left(S, 0^{-}\right)=S\left[B_{1}(S)-B_{2}(S)\right]=\frac{1}{\mu} \quad a^{s} \bar{u}^{s}(s)$

But

$$
\begin{aligned}
& -S A_{1}(S) \sin \pi / 2 S+S B_{1}(S) \cos \pi / 2 S=\frac{1}{\mu} T l^{S} ; 0 \leq \theta \leq \pi / 2 \\
& S A_{2}(S) \sin \pi / 2 S+S B_{2}(S) \cos \pi / 2 S=\frac{1}{\mu} Q h^{s} ; \quad-\pi / 2 \leq \theta \leq 0
\end{aligned}
$$

This gives

$$
B_{1}(S) \frac{T l^{s}+\mu S a^{S} \bar{V}(S) \sin \pi / 2 S}{\mu S \cos \pi / 2 S}
$$

Similarly

Hence,

$$
B_{2}(S) \frac{Q h^{s}-\mu S a^{s} \bar{V}(S) \sin \pi / 2 S}{\mu S \cos \pi / 2 S}
$$

$$
S\left[B_{1}(S)-B_{2}(S)\right]=\frac{T l^{s}+\mu S a^{s} \bar{V}(S) \sin \pi / 2 s}{\mu S \cos \pi / 2 S}
$$




$$
\begin{aligned}
& -\frac{\left[Q h^{s}-\mu s a^{s} \bar{V}(s) \sin \pi / 2 s\right]}{\mu s \cos \pi / 2 s} \\
& \frac{1}{\mu} a^{s} \tilde{u}(S)=\frac{a^{s}}{u}\left[\frac{T\left(\frac{l}{a}\right)^{s}-Q\left(\frac{h}{a}\right)^{s}}{\cos \pi / 2 s}+\frac{2 \mu s \tilde{V}(s) \sin \pi / 2 s}{\cos \sin \pi / 2 s}\right.
\end{aligned}
$$

Hence,

$$
\widetilde{U}(S)=\frac{T\left(\frac{l}{a}\right)^{s}-Q\left(\frac{h}{a}\right)^{s}}{\cos \pi / 2 S}+\frac{2 \mu s \bar{V}(S) \sin \pi / 2 s}{\cos \pi / 2 S}
$$

From (7a) and (14) we see that the half known function $\tilde{u}(s)$ is analytic in the left half plane

Res $>\lambda-1$. Hence, we denote it by $\tilde{u}(s)$ and from (7b) and (11b) it is seen that $\tilde{V}(s)$ is analytic in the left plance Res $<0$ we therefore, denote it by $\tilde{V}(s)$.

Thus;

$$
\tilde{u}_{+}(s)=\frac{2 \mu S \sin \pi / 2 s}{\cos \pi / 2 s}\left[\tilde{V}(s)+\frac{E(s)}{S \cos \pi / 2(s-1)}\right]
$$

Where,

$$
E(s)=\frac{T}{2 \mu}\left(\frac{l}{a}\right)^{s}-\frac{Q}{2 \mu}\left(\frac{h}{a}\right)^{s}
$$

\section{Solution Of The Wiener - Hoph Equation}

To achieve the decomposition of the trigonometric function, we introduce the infinite product theorem [5]

$$
\sin \pi / 2 s=\pi / 2 s \prod_{n=1}^{\infty}\left[1-\left(\frac{s}{2 n}\right)^{2}\right]
$$

Therefore,

$$
\frac{2 \mu S \sin \pi / 2 s}{\cos \pi / 2 s}=\frac{4 u S \sin ^{2} \pi / 2 s}{\sin \pi s}
$$

Leads to

$$
\frac{4 u S \sin ^{2} \pi / 2 s}{\sin \pi s}=\frac{N_{-}(s)}{N_{+}(s)}
$$

Substituting into (28) we have,

$$
\tilde{\mu}_{+}(s)=\frac{N_{-}(s)}{N_{+}(s)}\left[\tilde{V}(s)+\frac{E(s)}{S \cos \pi / 2(s-1)}\right]
$$

We obtain

$$
\tilde{\mu}_{+}(s) N_{+}(s)=N_{-}(s) \tilde{V}(s)+\frac{N_{-}(s) E(s)}{S \cos \pi / 2(s-1)}
$$

The mixed term in (34) is decomposed into a sum using the mittag-leffler's theorem (6)

$$
\frac{N_{-}(s)}{S} \cdot \frac{E(s)}{\cos \pi / 2 s(s-1)}=M_{+}(s)+M_{-}(s)
$$

From the relationship between Gamma function and infinite product

And

$$
\begin{aligned}
& \frac{4 u s \sin ^{2} \pi / 2 s}{\sin \pi s}=\left[\frac{\mu s^{2} \pi\left[\prod_{n=1}^{\infty}\left(1-\frac{s}{2 n}\right)^{2}\right]}{\prod_{n=1}^{\infty}\left(1-\frac{s}{n}\right)}\right]\left[\frac{\left[\prod_{n=1}^{\infty}\left(1+\frac{s}{2 n}\right)\right]^{2}}{\prod_{n=1}^{\infty}\left(1+\frac{s}{n}\right)}\right] \\
& N_{-}(s)=\frac{u s^{2} \pi\left[\prod_{n=1}^{\infty}\left(1-\frac{s}{2 n}\right)\right]^{2}}{\prod_{n=1}^{\infty}\left(1-\frac{s}{n}\right)} e^{\chi s}
\end{aligned}
$$

$$
N_{+}(s)=\frac{\left[\prod_{n=1}^{\infty}\left(1-\frac{s}{n}\right)\right] e^{\chi s}}{\left[\prod_{n=1}^{\infty}\left(1+\frac{s}{2 n}\right)\right]^{2}}
$$

Where $\chi$ will be chosen so that $N_{-}(s)$ and $N_{+}(s)$ have algebraic behavior as $|s| \rightarrow \infty$. On further decomposition, from mittag-leffler's theeorem 
We have

$$
\frac{\sec \pi / 2(s-1)=\frac{4}{\pi} \sum_{n=1}^{\infty}(-1)^{n+1}(2 n-1)}{(2 n-1)^{2}-(s-1)^{2}}
$$

$$
M_{+}(s)=\frac{2}{\pi} \sum_{n=1}^{\infty}(-1)^{n}\left[\frac{N_{-}(s) E(s) N_{-}\left(-\xi_{n}\right) E\left(-\xi_{n}\right)}{s-\xi_{n}}-\frac{N_{-}(s) E(s)}{s-\xi_{n-1}}\right]
$$

And

$$
M_{-}(s)=\frac{2}{\pi} \sum_{n=1}^{\infty}(-1)^{n} \frac{N_{-}\left(-\varepsilon_{n}\right) E\left(-\varepsilon_{n}\right)}{s-\varepsilon_{n}}
$$

We have from the decomposition that

$$
N_{+}(s) \tilde{u}_{+}(s)=\tilde{V}_{-}(s) N_{-}(s)+M_{+}(s)+M_{-}(s)
$$

Hence by analytic continuation

Now

$$
N_{+}(s) \tilde{u}_{+}(s)-M_{+}(s)=\tilde{V}_{-}(s) N_{-}(s)+M_{-}(s)=C
$$

Considering the behavior of;

$$
\tilde{V}_{-}(s) N_{-}(s)+M_{-}(s)=C
$$

We get

Hence $C=M_{-}(0)$

$$
M_{-}(s) ; \quad N_{-}(s) \text { and } \tilde{V}_{-}(s) \text { at } s=0
$$

$$
N_{-}(s)=0 ; \quad M_{-}(s) \neq 0
$$

We then have

$$
\tilde{V}_{-}(s) N_{-}(s)+M_{-}(s)=M_{-}(0)
$$

This gives

$$
\tilde{V}_{-}(s)=\frac{M_{-}(0)-M_{-}(s)}{N_{-}(s)}
$$

And

$$
\tilde{u}_{-}(s)=\frac{M_{+}(0)-M_{+}(s)}{N_{+}(s)}
$$

We have for $\quad 0 \leq \theta \leq \frac{\pi}{2}$

\section{The Mellin Transform Formular}

$$
\begin{gathered}
\bar{W}(S, \theta)=\frac{a^{s} \bar{V}(S) \cos \left(\frac{\pi}{2}-\theta\right) s}{\cos \pi / 2^{s}}+\frac{T l^{s} \sin \theta s}{\mu s \cos \pi / 2^{s}} \\
=a^{s}\left[\frac{T\left(\frac{l}{a}\right)^{s} \sin \theta s}{\mu s \cos \pi / 2^{s}}+\frac{M_{-}(0)-M_{-}(s)}{N_{-}(s)} \frac{\cos \left(\frac{\pi}{2}-\theta\right) s}{\cos \pi / 2^{s}}\right]
\end{gathered}
$$

And for $\pi / 2 \leq \theta \leq 0$; we have

$$
\bar{W}(S, \theta)=a^{s} \frac{Q\left(\frac{h}{a}\right)^{s} \sin \theta s}{\mu \mathrm{cos} \pi / 2^{s}}+\left(\frac{M_{-}(0)-M_{-}(s)}{N_{-}(s)}\right) \frac{\cos \left(\frac{\pi}{2}+\theta\right) s}{\cos \pi / 2^{s}}
$$

The inversion integral gives the displacement sought for as

Hence for $\quad 0 \leq r \leq a ; \quad 0 \leq \theta \leq \pi / 2$

$$
W(S, \theta)=\frac{1}{2 \pi i} \int_{c-i \infty}^{c+i \infty} \bar{W}(s, \theta) r^{-s} d s
$$

$$
W(S, \theta)=\frac{1}{2 \pi i} \int_{c-i \infty}^{c+i \infty}\left[\frac{T\left(\frac{l}{a}\right)^{s} \sin \theta s}{\mu s \cos \pi / 2 s}+\left(\frac{M_{-}(0)-M_{-}(s)}{N_{-}(s)}\right) \frac{\cos \left(\frac{\pi}{2}-\theta\right) s}{\cos \pi / 2 s}\right]\left(\frac{r}{a}\right)^{s} d s
$$

And for $0 \leq r \leq a ;-\pi / 2 \leq \theta \leq 0$

$$
W(r, 0)=\frac{1}{2 \pi i} \int_{c+i \infty}^{c+i \infty}\left[\frac{Q\left(\frac{h}{a}\right) \sin \theta s}{\mu \mathrm{cos} \pi / 2 s}+\frac{M_{+}(0)-M_{-}(s)}{N_{-}(s)} \frac{\cos \left(\frac{\pi}{2}+\theta\right) s}{\cos \pi / 2 s}\right]\left(\frac{r}{a}\right) d s
$$

For $C>$ Res; $\lambda-1$ Res $s<0: 0<\lambda<1 / 2$ 
To evaluate the inversion integral (51) and (52). The singularities of $\cos \pi / 2 s$ are all simple and are located at $s= \pm(2 n-1)$ for all $n \in N$. We use the residue theorem to obtain a closed form solution of the displacement as

For $0 \leq r \leq a ; \quad 0 \leq \theta \leq \frac{\pi}{2}$

$$
\begin{aligned}
W(r, \theta)=\frac{2}{\mu \pi} \sum_{n=1}^{\infty}(-1)^{n}\left[T\left(\frac{l}{a}\right)^{1-2 n} \frac{\sin (1-2 n) \theta}{1-2 n}+\mu \frac{M_{-}(0)-M_{-}(1-2 n)}{N_{-}(1-2 n)}\right. \\
\left.+\mu\left(\frac{M_{-}(0)-M_{-}(1-2 n)}{N_{-}(1-2 n)}\right) \cos \left(\frac{\pi}{2}-\theta\right)(1-2 n)\right]\left(\frac{r}{a}\right)^{2 n-1}
\end{aligned}
$$

And for $0 \leq r \leq a ;-\frac{\pi}{2} \leq \theta \leq 0$

$$
\begin{aligned}
W(r, \theta)=\frac{2}{\mu \pi} \sum_{n=1}^{\infty}(-1)^{n}\left[Q\left(\frac{h}{a}\right)^{1-2 n} \frac{\sin (1-2 n) \theta}{1-2 n}\right. \\
\left.+\mu\left(\frac{M_{-}(0)-M_{-}(1-2 n)}{N_{-}(1-2 n)}\right) \cos \left(\frac{\pi}{2}+\theta\right)(1-2 n)\right]\left(\frac{r}{a}\right)^{2 n-1}
\end{aligned}
$$

At the inhomegeneity tip: The outer tip

As $r \rightarrow 0 ; n=1 ; s=1-2 n$, for $n=1 ; s=-1 \xi_{n}=2 n ; \xi_{1}=2$

The displacement will be;

$$
W(r, 0)=\frac{-2}{\mu \pi a}\left[T\left(\frac{a}{l}+\frac{1}{3}\right)\left[\frac{T}{2}\left(\frac{a}{l}\right)^{2}-\frac{Q}{2}\left(\frac{a}{h}\right)^{2}\right]\right] r \sin \theta
$$

For $0 \leq r \leq a ; \quad 0 \leq \theta \leq \frac{\pi}{2}$

$$
W(r, \theta)=\frac{-2}{\mu \pi a}\left[Q \frac{a}{h}+\frac{1}{3}\left[\frac{T}{2}\left(\frac{a}{l}\right)^{2}-\frac{Q}{2}\left(\frac{a}{h}\right)^{2}\right] r \sin \theta\right.
$$

The stresses at the outer tip $r \rightarrow 0$

$$
\begin{gathered}
\sigma_{\theta \mathrm{z}}=\left\{\begin{array}{l}
\frac{-2}{\pi a}\left[T\left(\frac{a}{l}+\frac{1}{3}\right)\left[\frac{T}{2}\left(\frac{a}{l}\right)^{2}-\frac{Q}{2}\left(\frac{a}{h}\right)^{2}\right] \cos \theta\right. \\
\frac{-2}{\pi a}\left[Q \frac{a}{h}+\frac{1}{3}\left[\frac{T}{2}\left(\frac{a}{\lambda}\right)^{2}-\frac{Q}{2}\left(\frac{a}{h}\right)^{2}\right] \cos \theta\right.
\end{array}\right. \\
\sigma_{0 \mathrm{z}}=\left\{\begin{array}{l}
\frac{-2}{\pi a}\left[T \frac{a}{l}+\frac{1}{3}\left[\frac{T}{2}\left(\frac{a}{\lambda}\right)^{2}-\frac{Q}{2}\left(\frac{a}{h}\right)^{2}\right] \sin \theta ; 0 \leq \theta \leq \frac{\pi}{2}\right. \\
\frac{-2}{\pi a}\left[Q \frac{a}{h}-\frac{1}{3}\left[\frac{T}{2}\left(\frac{a}{l}\right)^{2}-\frac{Q}{2}\left(\frac{a}{h}\right)^{2}\right] \sin \theta ;-\frac{\pi}{2} \leq \theta\right.
\end{array}\right.
\end{gathered}
$$

At the inhomogeneity tip, the inner tip

The displacement is

$$
\begin{aligned}
& r \rightarrow a \text { and } \theta \rightarrow 0 ; \quad \rho \rightarrow 0 \\
& \left\{\begin{array}{l}
r \cos \theta=a+\rho \cos \psi \\
r \sin \theta=\rho \sin \psi \\
\frac{r}{a}=1+\frac{\rho}{a} \cos \psi+Q\left(\frac{\rho}{a}\right)
\end{array}\right.
\end{aligned}
$$

$$
W(\rho, \psi)=C_{0}-\frac{1}{\mu}\left(\frac{1}{\sqrt{4 \pi a}} M_{-}(0)\right)\left(\frac{2 \rho}{\pi}\right)^{\frac{1}{2}} \cos \frac{\psi}{2}
$$

For $0 \leq \theta \leq \frac{\pi}{2}$

Similarly

The stress components

$$
\begin{aligned}
& W(\rho, \psi)=d_{0}-\frac{1}{\mu}\left(\frac{1}{\sqrt{4 \pi a}} M_{-}(0)\right)\left(\frac{2 \rho}{\pi}\right)^{\frac{1}{2}} \cos \frac{\psi}{2} \\
& i \text { f or } \frac{-\pi}{2} \leq \theta \leq 0
\end{aligned}
$$

$\sigma_{\psi z}(\rho, \psi) ; \quad \sigma_{\rho z}(\rho, \psi)$ near the crack tip are given by 
This gives

$$
\sigma_{\psi z}(\rho, \psi)=\mu \frac{1}{\rho} \frac{\partial W}{\partial \psi}(\rho, \psi)
$$

$$
\begin{gathered}
\sigma_{\psi z}(\rho, \psi)=\frac{\mu}{\rho} \cdot \frac{1}{2 \mu}\left(\frac{1}{\sqrt{4 \pi a}} M_{-}(0)\right)\left(\frac{2}{\pi}\right)^{1 / 2} \rho^{\frac{1}{2}} \sin \frac{\psi}{2} \\
=\frac{1}{\sqrt{2 \pi \rho}}\left(\frac{1}{\sqrt{4 \pi a}} M_{-}(0)\right) \sin \frac{\psi}{2} \\
\sigma_{\rho z}(\rho, \psi)=\mu \frac{\partial W}{\partial \rho}(\rho, \psi) \\
=-\frac{1}{2}\left(\frac{1}{\sqrt{4 \pi a}} M_{-}(0)\right)\left(\frac{2}{\pi}\right)^{1 / 2} \rho^{\frac{1}{2}} \cos \frac{\psi}{2} \\
=-\frac{1}{\sqrt{2 \pi \rho}}\left(\frac{1}{\sqrt{4 \pi a}} M_{-}(0)\right) \cos \frac{\psi}{2}
\end{gathered}
$$

The stress intensity factor is

$$
K_{I I I}=\frac{M_{-}(0)}{\sqrt{4 \pi a}}
$$

This gives

$$
W(\rho, \psi)=C_{0}-\frac{1}{\mu} K_{I I I}\left(\frac{2 \rho}{\mu}\right)^{1 / 2} \cos \frac{\psi}{2} \text { for } 0 \leq \theta \leq \pi / 2
$$

And

$$
\begin{aligned}
& W(\rho, \psi)=d_{0}-\frac{1}{\mu} K_{I I I}\left(\frac{2 \rho}{\mu}\right)^{1 / 2} \cos \frac{\psi}{2} \text { for }-\pi / 2 \leq \theta \leq 0 \\
& \sigma_{\psi z}(\rho, \psi)=\frac{1}{\sqrt{2 \pi \rho}} K_{I I I} \sin \frac{\psi}{2} \\
& \sigma_{\psi z}(\rho, \psi)=\frac{1}{\sqrt{2 \pi \rho}} K_{I I I} \cos \frac{\psi}{2}
\end{aligned}
$$

V. Nalysis Of The Mode Iii Stress Intensity Factor

The standard form of the displacement is given by;

Where

$$
\begin{aligned}
& W(\rho, \psi)=C_{0}-\frac{1}{\mu} K_{I I I}\left(\frac{2 \rho}{\mu}\right)^{1 / 2} \cos \frac{\psi}{2} \text { for } 0 \leq \theta \leq \pi / 2 \\
& W(\rho, \psi)=d_{0}-\frac{1}{\mu} K_{I I I}\left(\frac{2 \rho}{\mu}\right)^{1 / 2} \cos \frac{\psi}{2} \text { for }-\pi / 2 \leq \theta \leq 0 \\
& \sigma \psi z(\rho, \psi)=\frac{1}{\sqrt{2 \pi \rho}} K_{I I I} \sin \frac{\psi}{2} \\
& \sigma \psi z(\rho, \psi)=\frac{1}{\sqrt{2 \pi \rho}} K_{I I I} \cos \frac{\psi}{2}
\end{aligned}
$$

$$
\begin{aligned}
& K_{I I I}=\frac{M_{-}(0)}{4 \pi a} \\
& M_{-}(0)=\frac{2}{\pi} \sum_{n=1}^{\infty} \frac{(-1)^{n} N\left(-\xi_{n}\right) E\left(-\xi_{n}\right)}{-\xi_{n}} \\
& N-\left(-\xi_{n}\right)=\frac{4 \mu \pi \xi_{n}\left\lceil\left(\xi_{n}\right)\right.}{\left[\Gamma\left(\frac{\xi_{n}}{2}\right)\right]^{2}} \varrho^{-\psi \xi_{n}} \\
& E\left(-\xi_{n}\right)=\left(\frac{1}{2 \mu}\right)\left(\frac{l}{a}\right)^{-\xi_{n}}-\frac{Q}{2 \mu}\left(\frac{h}{a}\right)^{-\xi_{n}}
\end{aligned}
$$




$$
\begin{gathered}
M_{-}(0)=\frac{2}{\pi} \sum_{n=1}^{\infty}(-1)^{n+1} \frac{\left[\left(\xi_{n}\right) \varrho^{-\psi \xi_{n}}\left[T-\xi_{n}\right]-Q\left(\frac{h}{a}\right)^{-\xi_{n}}\right.}{\left[\Gamma\left(\frac{\xi_{n}}{2}\right)\right]^{2}} \\
\text { for a "smaller than both land h, we have } \\
K_{I I I}(\rho, \psi)=\frac{4}{\sqrt{4 \delta a}} \frac{1}{2^{2}} \frac{\Gamma(2)}{[\Gamma(1)]^{2}}\left[T\left(\frac{a}{l}\right)^{2}-Q\left(\frac{a}{h}\right)^{2}\right] \\
K_{I I I}(\rho, \psi)=\frac{1}{\sqrt{4 \pi a}}\left[T\left(\frac{a}{l}\right)^{2}-Q\left(\frac{a}{h}\right)^{2}\right]
\end{gathered}
$$

For the case of equilibrating loading

$$
Q=-T \text { and } l=h
$$

We have

$$
K_{I I I}(\rho, \psi)=\frac{T}{\sqrt{\pi a}}\left(\frac{a}{l}\right)^{2}
$$

Denote by $K_{I I I}^{0}$ the stress intensity factor for the case of a crack in an elastic half plane under remote loading by

$$
K_{I I I}^{0}=\frac{\sqrt{2}}{\sqrt{\pi a}} T=\sqrt{\frac{2}{\pi a}} T
$$

We have;

$$
K_{I I I}(\rho, \psi)=\frac{1}{\sqrt{2}} \quad K_{I I I}^{0}\left(\frac{a}{l}\right)^{2}
$$

Defining $K_{I I I}{ }^{N O R}$; -Normalized stress intensity factor as

We have

$$
K_{I I I}^{N O R} ;=\frac{K_{I I I}}{K_{I I I}{ }^{0}}
$$

The strain energy $U$ stored in the matrix is given by

$$
K_{I I I}^{N O R}=\frac{1}{\sqrt{2}}\left(\frac{a}{l}\right)^{2}
$$

$$
\begin{gathered}
\bigcup=\frac{1}{2 \mu} \int_{1}\left[{\sigma_{\rho z}}^{2}+{\sigma_{\psi z}}^{2}\right] d F \\
=\frac{1}{2 \mu}-\frac{1}{2 \pi \rho} K_{I I I}^{2} \cdot 2 \pi \rho \\
=\frac{1}{2 \mu} K_{I I I}^{2}
\end{gathered}
$$

This gives the strain energy for equilibrating loading as

$$
\bigcup=\frac{1}{2 \mu} \frac{T^{2}}{\pi a}\left(\frac{a}{l}\right)^{4}
$$

\section{Conclusion}

A rigid line inhomogeneity embedded in an isotropic homogenuous material introduced in the region

$$
y=0 ; 0 \leq x \leq a ;-\infty<z<\infty
$$

and under anti-plane shear has been studied. The closed form solution obtained by the wiener-Hoph technique has been analyzed. The mode III stress intensity factor $K_{I I I}$ has been obtained. The analysis showed that Microcrack will ensure at the inner tip of the inhomogeneity; since the stresses showed square root singularity at the inner tip.

The energy release rate was also computed and found to be

$$
\frac{1}{2 \mu} K_{I I I}^{2}
$$


Figures

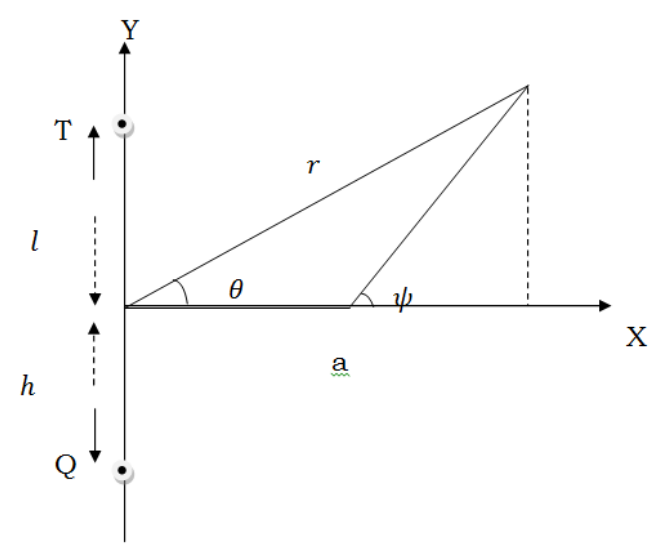

Fig. I: The problem

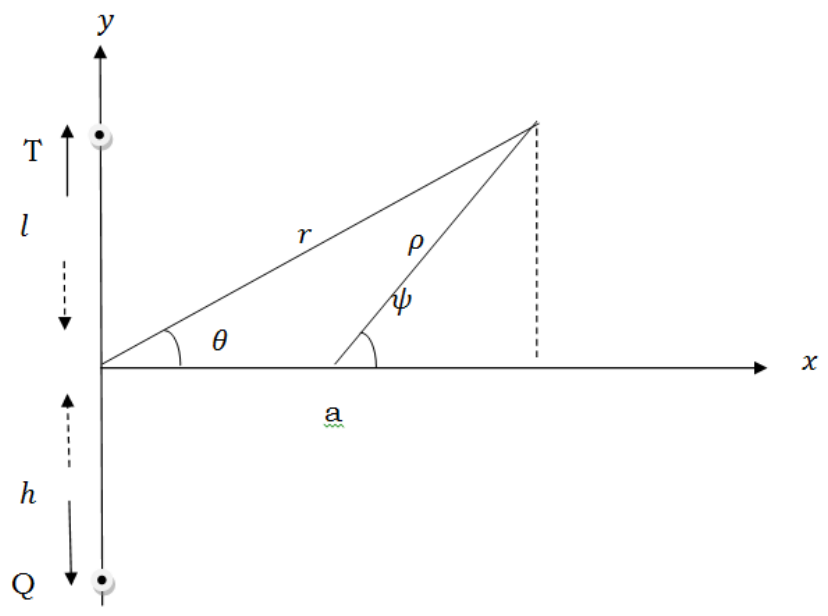

Fig. 2: Geometry of the problem

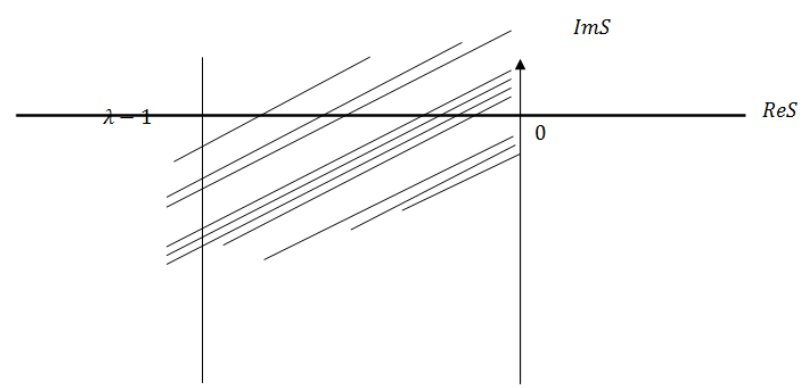

Fig. 3: Fundamental strip

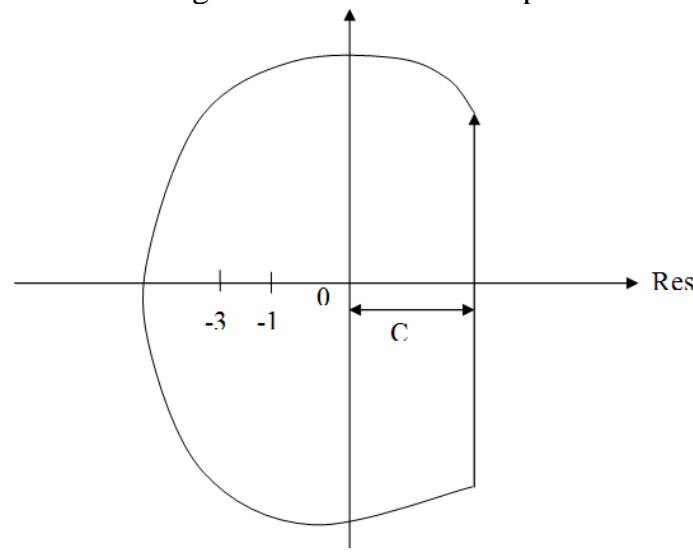

Fig. 4: Contour used in the inversion of the equations. 


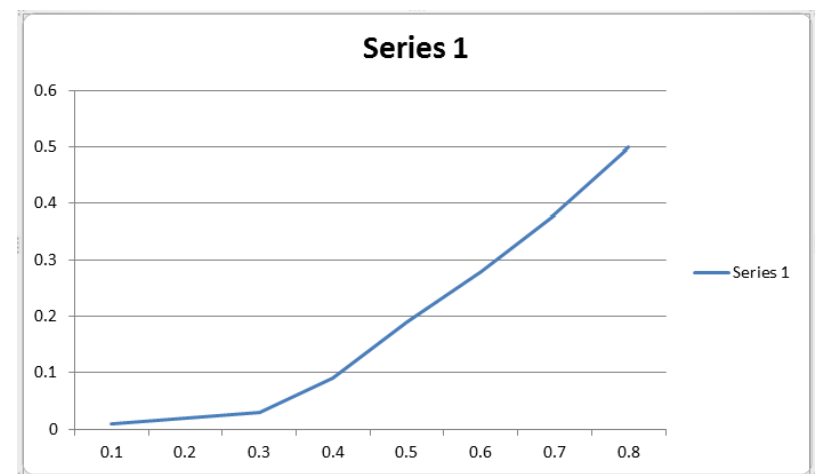

Fig. 5: Comparism of normalized stress intensity factor and $\frac{a}{l}$

\section{References}

[1] Z. M Xiao and H. Fan, Micro Crack initiation at tip of a rigid inhomogeneity. Journal of fracture Vol. 83: Pp 1-9, 1996

[2] J. Dundurs and X. Markenscoff: A Green's function formulation of anti-cracks and their interaction with load induced singularities. ASME journal of Applied Mechanics Vol. 56, Pp. 550-555, 1989

[3] H. Tada, P.C. Paris and G. R. Trwin. Stress intensity hand book. Del. Research corporation. Heller town pennsyvania 1993.

[4] P. C Paris and G. C. Sil, Stress analysis of cracks. Symposium on fracture Toughness, Testing and its application. ASTM Special Technical publication 381; Pp. 30-83; 1965

[5] M.G. Arfken: Mathematical methods for physicists Academic press Inc. New York. 1966.

[6] R. V. Churchill: Complex variables and applications $2^{\text {nd }}$ edition, McGraw-Hill, New York 1960. 